\title{
Spontaneous Uterine Perforation of Choriocarcinoma with Negative Beta-Human Chorionic Gonadotropin after Chemotherapy
}

\author{
Chuan Xie Lan Zheng Zheng-Yu Li Xia Zhao \\ Department of Gynecology and Obstetrics, West China Second Hospital, Sichuan University, Chengdu, PR China
}

\section{Key Words}

Choriocarcinoma - Uterine perforation - Human chorionic gonadotropin $\cdot$ Hysterectomy

\begin{abstract}
Objective: To report an extremely rare case of spontaneous uterine perforation of choriocarcinoma with negative betahuman chorionic gonadotropin ( $\beta$-hCG) post-chemotherapy. Clinical Presentation and Intervention: We present a 35-year-old choriocarcinoma patient whose serial serum $\beta$ hCG levels following a fifth course of chemotherapy had been within the normal range, but who developed spontaneous uterine perforation with intra-abdominal hemorrhage after eight courses of combined chemotherapy. The patient then underwent an emergency hysterectomy and survived. Conclusion: Patients with persistent focus of disease in the uterus might experience uterine perforation even after adequate chemotherapy, and therefore, the follow-up for patients after chemotherapy is very important.
\end{abstract}

Copyright $\odot 2011$ S. Karger AG, Basel

\section{Introduction}

Choriocarcinoma is a type of trophoblastic tumor, associated with aggressive biological behavior including local invasion of the uterus and metastasis to distant sites such as the lung and brain. The disease can often be cured by cytotoxic chemotherapy. Choriocarcinoma affects 1:40,000 pregnancies and 1:40 hydatidiform moles. However, choriocarcinoma presenting as spontaneous uterine perforation with intra-abdominal hemorrhage is extremely rare [1].

In this report, we describe a woman with spontaneous uterine perforation secondary to choriocarcinoma 3 months after $\beta$-human chorionic gonadotropin $(\beta$-hCG) normalized, who survived following emergency laparotomy and hysterectomy.

\section{Case Report}

A 35-year-old woman, gravida II, para I, abortion I, was admitted to our hospital with suspected choriocarcinoma. She had previously presented to a local hospital complaining of intermittent lower abdominal pain and irregular vaginal bleeding and was diagnosed with a hydatidiform mole. Curettage of the uterine cavity was then performed without pathological examination and follow-up for $\beta$-hCG. Nine months later, the patient presented again to hospital with irregular vaginal bleeding. The patient was admitted for further management of persistent trophoblastic disease.

On admission, bimanual examination revealed an enlarged uterus of $8-10$ weeks gestation. Her serum $\beta$-hCG level was $88,994.5 \mathrm{mIU} / \mathrm{ml}$, and transvaginal ultrasound revealed a complex mass in the uterus measuring $10 \times 7 \times 11 \mathrm{~cm}$. Chest radiography showed bilateral multiple pulmonary nodules confirmed by computed tomography without evidence of brain or liver metastases.

\section{KARGER}

Fax +41613061234

E-Mail karger@karger.ch

www.karger.com
(C) 2011 S. Karger AG, Basel

1011-7571/11/0206-0570\$38.00/0

Accessible online at:

www.karger.com/mpp
Prof. Xia Zhao

Department of Gynecology and Obstetrics

West China Second Hospital, Sichuan University

Chengdu 610041 (PR China)

Tel. +8628 8550 1633, E-Mail xia-zhao@126.com 
Fig. 1. Concentration of $\beta-\mathrm{hCG}$ during treatment. Serial serum $\beta$-hCG levels following the fifth course of chemotherapy were within the normal range. Dashed line $=$ normal range limit $(<5 \mathrm{mIU} / \mathrm{ml})$.

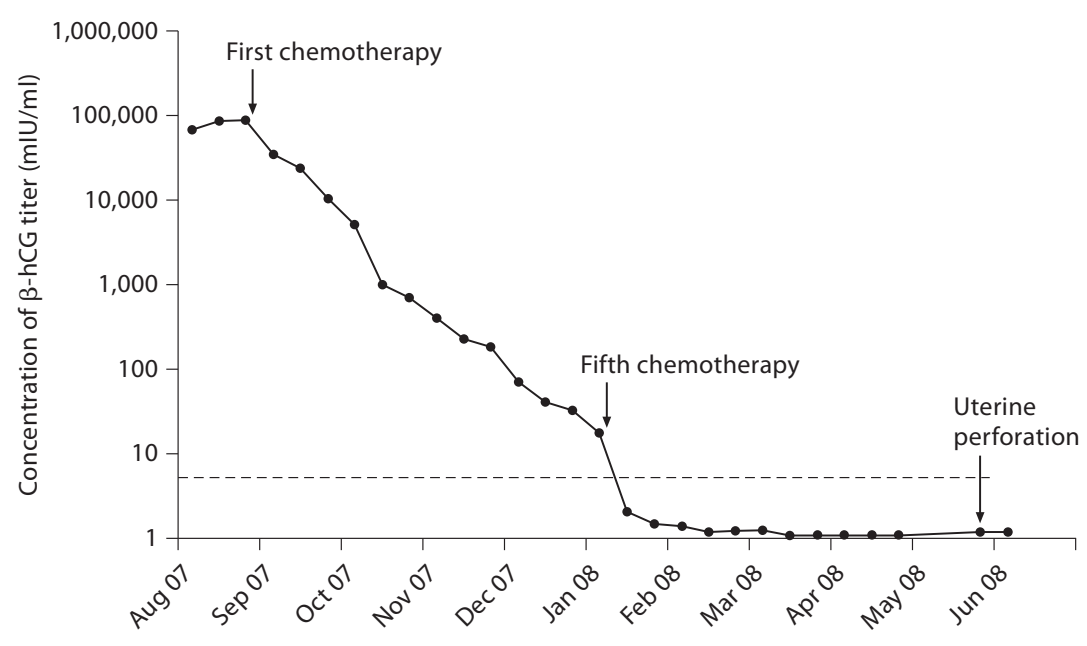

With the above findings, the patient was diagnosed with metastatic choriocarcinoma stage III FIGO in the WHO scoring system, at high-risk category. Combined chemotherapy was initiated as follows: 5-fluorouracil (5-FU) $24 \mathrm{mg} / \mathrm{kg}$.day and kengsengmycin (KSM) $6 \mu \mathrm{g} / \mathrm{kg} \cdot$ day intravenously from day 1 to day 8 . The above regimen was repeated at a 3-week interval. Serum $\beta$-hCG levels decreased in a time-dependent manner and normalized after five courses of chemotherapy (fig. 1). Serial chest X-ray also showed the gradually diminishing pulmonary infiltrates, which completely resolved after the fourth chemotherapy. Transvaginal ultrasound revealed that the intrauterine mass was $2.6 \mathrm{~cm}$ in diameter after the fifth chemotherapy. Because of refusal of surgical treatment by the patient, three additional courses of chemotherapy were performed as consolidation therapy. Serum $\beta$-hCG levels during the three additional courses remained normal, and ultrasound revealed no pronounced change in the size of carcinoma foci.

One month after the end of chemotherapy, the patient was admitted to the emergency room for acute lower abdominal pain and sudden vaginal bleeding. Abdominal ultrasound findings showed an enlarged uterus and blood clots in the pelvic cavity, and temporarily $\beta$-hCG was not obtained. On suspicion of uterine perforation secondary to choriocarcinoma, the patient underwent an emergency exploratory laparotomy. During the procedure, the uterus was macroscopically enlarged with one site of perforation measuring $2 \times 2 \mathrm{~cm}$ in the posterior wall of the uterus with active bleeding. There were approximately $600 \mathrm{ml}$ of clotted blood in the peritoneal cavity secondary to uterine perforation. Bilateral ovaries and salpinges were found to be normal. A total abdominal hysterectomy was performed. A macroscopic examination of the dissected uterus specimen showed that the entire uterine wall had been invaded by tumor tissue, and there were approximately $80 \mathrm{ml}$ of clotted blood within the uterine cavity. A dark red fleshy hemorrhagic and necrotic mass measuring $2.5 \times$ $2 \mathrm{~cm}$ in diameter was visible in the posterior wall of the uterus specimen. Following surgery, the level of $\beta$-hCG was within the normal range.

Uterine Perforation of Choriocarcinoma
Histopathologic examination revealed large amounts of degenerative trophoblast cells in the posterior uterine wall around which extensive hyaline change, focal calcification and foreign body giant cell reaction of smooth muscle were detected, which were in accordance with postchemotherapy changes of choriocarcinoma (fig. 2).

After 7 days' hospitalization, the patient was discharged with a follow-up plan for serial $\beta$-hCG measurement. At present, 17 months after surgery, the patient is well with normal levels of $\beta$-hCG and without signs of recurrence.

\section{Discussion}

Gestational choriocarcinoma arising from placental trophoblastic tissue is a malignant germ cell tumor that can be associated with any type of gestational event, most often a complete hydatidiform mole [2]. Early metastasis to distant sites, especially to the lungs, liver, and brain, is quite common.

It is widely accepted that patients with choriocarcinoma are best managed by stratifying their treatment according to the recognized adverse prognostic features. According to the FIGO 2000 scoring system, most patients with a score of 6 or less should be classified as lowrisk and treated with single-agent chemotherapy, while those with a score of 7 or more should be categorized as high-risk and combined chemotherapy initiated, such as $5-\mathrm{FU}+\mathrm{KSM}$ and EMA-CO regimes [3]. The patient in this report began with the 5-FU + KSM chemotherapy regime. Her serum $\beta$-hCG decreased from $88,994.5$ to 
Fig. 2. Morphology and microscopic features of the resected uterus (HE).
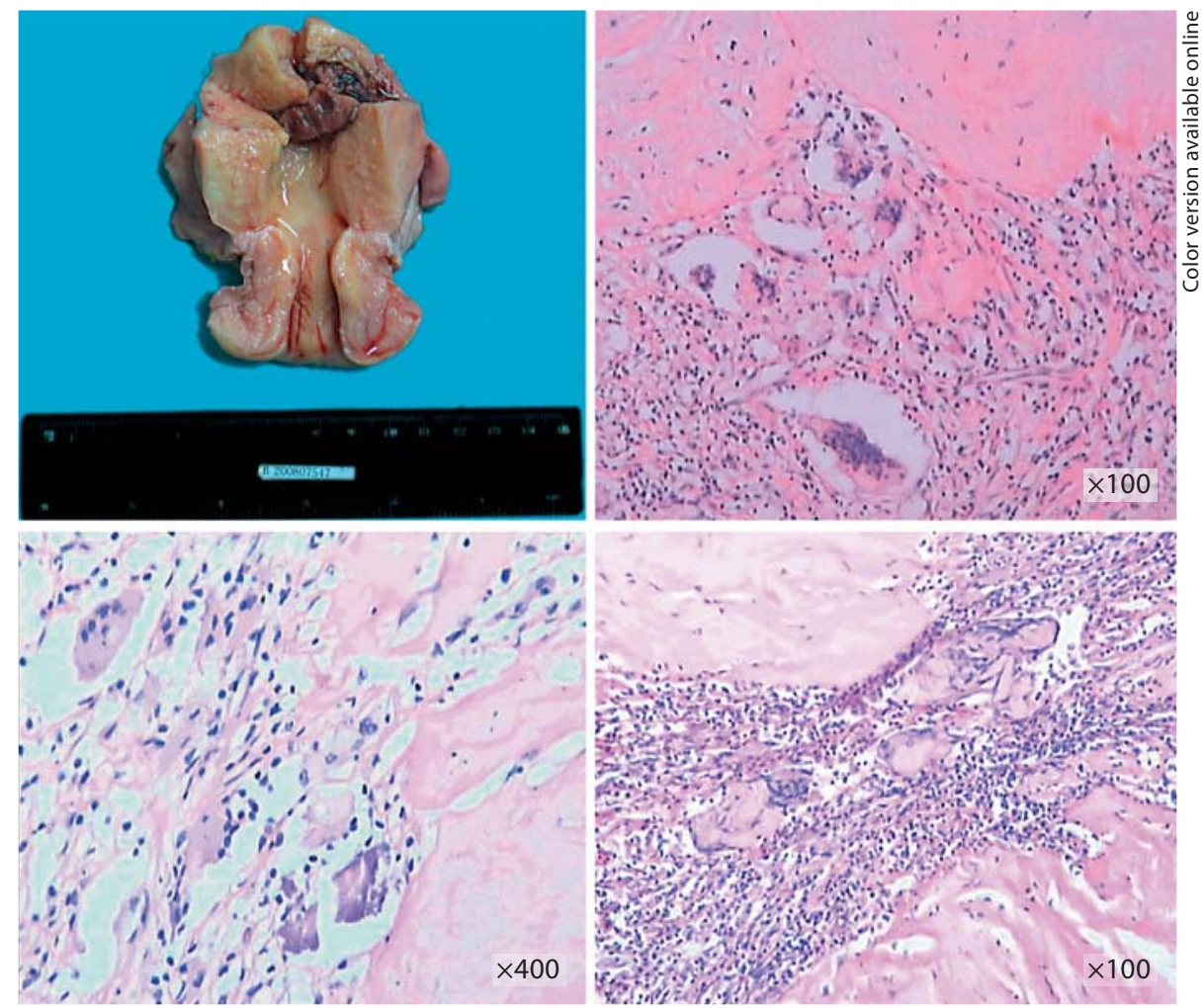

$10,351.8$ after the first course of chemotherapy and became negative after five courses (fig. 1). The falling of serum $\beta$-hCG level demonstrated the effectiveness of the multichemotherapy regime for this patient.

hCG secreted by cytotrophoblastic cells is composed of two polypeptide subunits: $\alpha$ and $\beta$, and the $\beta$ chain is unique to hCG [4]. Serum $\beta$-hCG is generally intact during the first trimester of healthy pregnancy. However, many other forms of $\beta$-hCG, including $\beta$-core, free- $\beta$ hCG, or nicked free- $\beta$, can exist in cancers like choriocarcinoma, which may be the reason why some commercial assays fail to detect some cancer-specific subtypes [5]. In our case, several different commercial assays were applied to eliminate a possible false-negative result.

During chemotherapy treatment, $\beta$-hCG is a useful tumor marker to monitor the patient's response to treatment and assess the focus of disease in the uterus. In our case, the pulmonary lesions disappeared after four courses of chemotherapy and serial weekly $\beta$-hCG was negative following the fifth course (fig. 1). The size of disease foci localized in the patient's posterior uterine wall diminished from 7 to $2.6 \mathrm{~cm}$ in diameter during the five courses of chemotherapy, but it persisted during the three additional courses of consolidation chemotherapy. Previ- ous studies have postulated that when hCG titers become undetectable, chemotherapy can be discontinued after two to three additional courses $[6,7]$. As the concentration of serum $\beta$-hCG reflects the amount of viable trophoblastic tissue, a negative $\beta$-hCG result may clinically reflect the absence of invasive cytotrophoblasts that could invade normal myometrium and cause uterine perforation. However, in our case, the patient developed spontaneous uterine perforation after 3 months with negative $\beta$-hCG. According to some studies, tumors like choriocarcinoma are hypervascular and can cause uterine perforation due to myometrial invasion $[8,9]$. Trophoblastic cells can invade blood vessels in the uterus. The damaged blood vessels may thrombose and result in single or multiple infarctions. Blood vessels may also develop tumorous aneurysms and bleed into the tissues surrounding the tumor or tumor tissue $[1,10]$. For this case, the exact cause of spontaneous uterine perforation of choriocarcinoma remains unclear. A possible explanation is that the effective treatment, which killed off the trophoblastic tissue, resulted in an area of necrosis large enough to cause a spontaneous perforation to develop in the uterus. It is difficult to determine whether choriocarcinoma cells could be eradicated completely when a negative $\beta$-hCG 
titer was achieved after chemotherapy. Surgery might be considered to be an effective approach to prevent the occurrence of uterine perforation for persisting focus in the uterus even when a negative postchemotherapy $\beta$-hCG titer has been achieved.

Hysterectomy is generally recommended when severe active intra-abdominal bleeding from uterine perforation of choriocarcinoma is found in urgent exploratory laparotomy [11]. However, for those young patients who desire pregnancy, conservative surgery in the form of a localized resection to remove the residual tumor from the uterus, followed by uterine reconstruction, would be preferred when the lesion is confined to the uterus without metastasis [12]. But severe intraperitoneal bleeding from choriocarcinoma may occasionally necessitate hysterectomy as an emergency procedure. In addition, hysterectomy is still an option in treating localized chemo-resistant choriocarcinoma if metastatic disease is carefully ruled out $[13,14]$.
Uterine perforation secondary to choriocarcinoma is rare, and it is even rarer when hCG becomes negative for 3 months after chemotherapy. To the best of our knowledge, no similar cases have been reported previously.

\section{Conclusion}

A negative hCG titer does not always indicate the disappearance of viable choriocarcinoma cells and the complete cure of choriocarcinoma, and may instead relate to the sensitivity of the assay. It is suggested that patients with persistent focus of disease in the uterus may experience uterine perforation even after adequate chemotherapy, and the follow-up for patients after chemotherapy is very important. When patients develop such emergencies as acute intraperitoneal bleeding, abdominal pain or shock, indicating the possibility of uterine perforation, surgical treatment might be necessary and optimal, and hysterectomy is generally recommended for most cases.

\section{References}

$>1$ Liberis V, Bouchlariotou S, Ammari A, Psillaki A, Ntatidou M, Sivridis E, Dislian V, Koutlaki N: Acute abdomen as initial presentation of gestational choriocarcinoma. Arch Gynecol Obstet 2009;280:859-862.

$>2$ Soper JT: Gestational trophoblastic disease. Obstet Gynecol 2006;108:176-187.

$>3$ Kendall A, Gillmore R, Newlands E: Chemotherapy for trophoblastic disease: current standards. Expert Rev Anticancer Ther 2003;3:48-54.

$\checkmark 4$ Stenman UH, Alfthan H, Hotakainen K: Human chorionic gonadotropin in cancer. Clin Biochem 2004;37:549-561.

5 Seckl MJ, Sebire NJ, Berkowitz RS: Gestational trophoblastic disease. Lancet 2010; 376:717-729. $\checkmark 6$ Cohn DE, Herzog TJ: Gestational trophoblastic diseases: new standards for therapy. Curr Opin Oncol 2000;12:492-496.

7 Sasaki S: Management of gestational trophoblastic diseases in Japan: a review. Placenta 2003;24(suppl A):S28-S32.

8 Okamoto T, Nomura S, Nakanishi T, Yamada S, Tomoda Y: A case of uterine choriocarcinoma with spontaneous rupture twentythree years following the antecedent pregnancy. J Obstet Gynaecol Res 1997;23: 189-195.

$>9$ Ma Y, Xiang Y, Wan XR, Chen Y, Feng FZ, Lei CZ, Yang XY: The prognostic analysis of 123 postpartum choriocarcinoma cases. Int J Gynecol Cancer 2008;18:1097-1101.

10 Mas JL, Lamy C: Stroke in pregnancy and the puerperium. J Neurol 1998;245:305-313.
11 Pisal N, North C, Tidy J, Hancock B: Role of hysterectomy in management of gestational trophoblastic disease. Gynecol Oncol 2002; 87:190-192.

12 Behtash N, Ansari S, Sarvi F: Successful pregnancy after localized resection of perforated uterus in choriocarcinoma and a literature review. Int J Gynecol Cancer 2006;16: 445-448.

13 Lehman E, Gershenson DM, Burke TW, Levenback C, Silva EG, Morris M: Salvage surgery for chemorefractory gestational trophoblastic disease. J Clin Oncol 1994;12:27372742.

14 Lurain JR: High-risk metastatic gestational trophoblastic tumors: current management. J Reprod Med 1994;39:217-222. 